\title{
A Culture in Transition: Paramedic Experiences with Community Referral Programs
}

\author{
Madison Brydges, BSc, MA*; Chris Spearen, $\mathrm{BSc}^{\dagger}$; Arija Birze, $\mathrm{PhD}(\mathrm{c})^{\ddagger}$; Walter Tavares, $\mathrm{PhD}^{* \S \uparrow}$
}

\section{ABSTRACT}

Objectives: As an aging population continues to place strain on the health care system, many older adults are living with unmet social and medical needs. In response, Emergency Medical Services (EMS) have initiated programs that encourage paramedics to refer patients in need to community based support services. This qualitative study explores frontline paramedic experiences with referral programs to identify opportunities and challenges in their practice.

Methods: This study used an intepretivist qualitative study design involving interviews of frontline paramedics employed in a region where referral programs were in place. Interviews were semi-structured and one-on-one. Data were transcribed verbatim and analyzed using inductive open coding throughout, then grouped to identify themes. Data collection and analysis were conducted simultaneously and flexibly until saturation.

Results: Twenty-three interviews were conducted representing 6 regions. When participating with referral programs the data revealed that frontline paramedics appear to experience (a) role confusion, (b) an inadequate knowledge base, (c) inadequate feedback, (d) undefined accountability, and (e) strong patient advocacy.

Conclusions: In a strained health care system, EMS and paramedics have an opportunity to better serve patients by initiating referrals for patients they encounter with unmet social and medical needs. However, referral programs face a number of challenges that, if left poorly addressed, may threaten the success of such programs.

\section{RÉSUMÉ}

Objectif : Comme le poids du vieillissement de la population ne cesse d'exercer des pressions sur le système de soins de santé, bon nombre de personnes âgées ont des besoins médicaux et sociaux non satisfaits. Devant cette situation, des services médicaux d'urgence (SMU) ont mis sur pied des programmes qui incitent les ambulanciers paramédicaux à diriger les patients dans le besoin vers des services communautaires de soutien. II s'agit d'une étude qualitative, qui visait à examiner l'expérience des programmes d'aiguillage des patients, vécue par les ambulanciers paramédicaux de première ligne, afin d'en cerner les possibilités et les difficultés d'application dans la pratique.

Méthode : Les auteurs ont adopté un plan d'étude qualitatif, réalisé selon une approche " interprétativiste ", qui consistait en des entrevues, semi-structurées et individuelles, avec des ambulanciers paramédicaux de première ligne, travaillant dans des régions où étaient appliqués les programmes d'aiguillage des patients. Les données ont d'abord été transcrites textuellement; puis analysées dans leur ensemble à l'aide d'un codage ouvert, inductif; et finalement groupées pour permettre aux chercheurs d'en dégager les grands thèmes. La collecte de données et l'analyse ont été réalisées en même temps et avec souplesse jusqu'à saturation.

Résultats : il y a eu 23 entrevues, concernant 6 régions. D'après les données recueillies sur les ambulanciers paramédicaux de première ligne, qui participaient aux programmes d'aiguillage des patients, il semblait y avoir : a) une confusion de rôle; b) une base insuffisante de connaissances; c) un manque de rétroaction; d) une obligation de répondre de ses actes non définie; (e) une forte empathie pour les patients.

Conclusions : Dans un système de soins de santé soumis à de fortes pressions, les SMU et les ambulanciers paramédicaux ont la possibilité de mieux rendre service aux patients qu'ils rencontrent en dirigeant ceux qui ont des besoins médicaux et sociaux non satisfaits vers les ressources appropriées. Toutefois, ces programmes connaissent un certain nombre de problèmes qui, s'ils sont négligés, risquent de nuire à leur réussite.

Keywords: paramedic, referral, culture, community paramedicine, qualitative

\section{INTRODUCTION}

The population of individuals over the age of 65 is expected to double in the next two decades. ${ }^{1}$ The majority will stay in their homes longer, either from personal choice or because of inadequate health care support. ${ }^{2}$ Many will require assistance with activities of

From the *McMaster University, Hamilton, ON; †York Region EMS, East Gwillimbury, ON; $¥$ University of Toronto Wilson Center, Toronto, ON; $\S$ Centennial College, Toronto, ON; and IParamedic Association of Canada, Ottawa, ON.

Correspondence to: Madison Brydges, Kenneth Taylor Hall, McMaster University, Room 226, 1280 Main Street W., Hamilton, ON, L8S 4M4; Email: madison.brydges@gmail.com 
daily living and in-home care to maintain their independence. $^{2}$ There is the risk that many of these issues will be detected late, managed poorly or not addressed, with the potential of perpetuating a difficult cycle of poor quality of life for the individual.

In Ontario, Canada, Community Care Access Centers (CCACs) provide individuals with access to government-funded home health care to mitigate these issues. These services include home-based nursing, nutrition and meal assistance, medication assistance, occupational therapy, and social support. ${ }^{2}$ However, there is evidence to suggest that the complexity of the system and the lack of a central access point means that residents remain uninformed about how to access or navigate these services independently. ${ }^{2}$

One such strategy to address this issue could involve engaging paramedics in community health care initiatives. $^{3}$ In many instances, individuals who initiate 911 calls often do not require urgent interventions or care in the $\mathrm{ED}$, but rather some level of long-term care directed at addressing their unmet social or medical needs. ${ }^{3}$ Paramedics have demonstrated capacity in identifying individuals at risk of deterioration in the home. ${ }^{4,5}$ As such, the paramedic community has developed, implemented, and continues to expand programs involving paramedics initiating referrals to community services (referred to as "community referrals by EMS (Emergency Medical Services)" or "CREMS"). Referral programs aim to promote the health and quality-of-life of seniors, improve their access to resources, and reduce 911-system activations and hospital visits. ${ }^{3}$

The purpose of this study was to explore all levels of paramedic interaction with referral programs. By asking paramedics to share their experiences with such programs, we aimed to increase our understanding of the ways in which paramedics experience and participate in community-based referral programs.

\section{METHODS}

\section{Study design}

An in-depth interview based, interpretivist ${ }^{6}$ qualitative study design was used to explore paramedic participation in referral programs. We took an interpretivist approach to understand the paramedics' experiences with the CREMS program. This is an appropriate epistemological framework for determining the meanings and lived experiences of the participants. Participants were interviewed via telephone using a semi-structured interview format conducted by the principal investigator. Consistent with an interpretive study design, ${ }^{6,7}$ we used open-ended, non-judgmental questions and probes to inquire more deeply where appropriate. Participants were asked to describe and reflect on their views and experience regarding referral programs.

An interview protocol and interview guide was used to assist the process and ensure consistency. Questions were generated through consensus among the research team, and then piloted for clarity, intent, potential biases, and appropriateness. Two pilot interviews were conducted with paramedics from an EMS with an existing referral program. Consistent with our approach, as new themes arose from the interview data, subsequent participants were asked additional questions related to emerging themes. ${ }^{6}$

\section{Sampling}

Following ethics approval from the Centennial College Research Ethics Board, we began with purposeful criterion sampling at the individual level. The inclusion criterion was: paramedics who were employed in a service that maintained a referral program and who identified themselves as having enrolled, or having the opportunity to enroll, patients in a referral program. Three EMS with CREMS programs and a professional EMS organization (Ontario Paramedic Association) distributed the study invitation via email. As all EMS services in Ontario with CREMS have a similar program as described above, we aimed to include participants from a variety of services, both male and female, a range of years of experience, method of referral (electronic or paper) and a range in the amount of reported referrals, including a participant who reported making no referrals (see Table 1).

Our sample size was based on thematic saturation to ensure that any emerging themes were sufficiently supported with substantial depth and scope. ${ }^{6,7}$ Based on our chosen methodology and research questions, as well as guidelines and recommendations provided by Creswell, ${ }^{6}$ we anticipated needing 20-30 in-depth interviews. Based on these recommendations, our analysis was conducted as interviews were completed, and rather than identifying a set number of interviews, we planned to use thematic saturation (i.e., the point at 
Table 1. Demographic information of study participants.

Demographic

Gender

Years of Experience

Estimated Number of Referral/years

Practice setting

Method of Referral to CCAC (electronic, paper referral, phone call)
Females: 8; Males: 15

Shortest: 1 year; Longest: 36 years; Mean: 12.8 years; Standard Deviation: 11.5 Lowest: 0; Highest: 30-40; Mean: 6; Standard Deviation: 6.7

Urban: 8; Suburban: 13; Rural: 2

Electronic referral: 12; Paper referral: 11 which no new information emerged from our interview data) as our end point. ${ }^{6,8}$ Due to initial difficulties contacting participants, we modified our sampling strategy to include snowball sampling. ${ }^{9}$ Contact with the initial group of key informants was achieved through existing contacts with paramedics from regional EMS.

\section{Data analysis}

Data analysis proceeded simultaneously with data collection, allowing the research team to identify emerging themes, use these themes to inform future interviews, and achieve saturation. ${ }^{6}$ This method of thematic saturation describes the point at which themes are fully accounted for, understood by, and agreed upon by the researchers, and where no new concepts or themes emerge from further interviews. ${ }^{8}$ Data analysis was conducted manually by the researchers and began with open coding, which allows the codes to emerge from the data. These codes were then organized into categories to reveal patterns and themes. ${ }^{6,9} \mathrm{Next}$, the categories were reviewed repeatedly, described using the collected and emerging data, and re-conceptualized until major themes emerged. Intensive, long-term involvement with the data leading to a thick description of the interviews further supported the analysis. ${ }^{6}$

To support trustworthiness in the interpretation of our data, transcripts were reviewed repeatedly to ensure they did not contain transcription errors. Next, data analysis was performed independently by the research team and any inconsistencies were resolved by joint review and discussion.

Clarifying the backgrounds of the research team is important for the participants and end-users of the data in qualitative research. ${ }^{6}$ Three members of the research team are employed by an EMS in southern Ontario as paramedics, educators or management (MB, WT, CS). Finally, another member of the research team $(\mathrm{AB})$ has a background in the sociology of education.

\section{RESULTS}

Data collection began in January 2013 and continued until April 2013. In total, 23 frontline paramedics from six EMS across Ontario participated in this study (see Table 1 for demographic information).

From the interviews, five themes emerged: (a) role confusion, (b) an inadequate knowledge base, (c) inadequate feedback, (d) undefined accountability, and (e) patient advocacy.

\section{Role confusion}

One of the major challenges for paramedics, and the most dominant theme, was that referral programs confronted paramedics with an alternative approach to patient care that was in conflict with larger workplace cultural beliefs grounded in emergency response. Many of the participants perceived their role as a paramedic to be defined by responding to emergency calls for help (i.e., consistent with their initial education and certification expectations), and referral programs represented a formal departure from that enduring view. Paramedics indicated that this change towards spending more time assessing and understanding patients' unmet long-term needs was in direct contrast with traditional values and requirements associated with EMS, such as identifying life threats and transporting all patients without delay (Table 2, quote 1a). Other indicators emerged from the data as well, such as peers having more influence over participants' participation in the program than performance expectations or standards of care. CREMS was rarely discussed among peers (compared to other aspects of the profession). Frustration was often expressed when others were unaware of the program (Table 2, quote $1 \mathrm{~b}$ ) or failed to participate (Table 2, quote 1c). Furthermore, there was discordance when discussing whether referral programs were part of a separate "community paramedicine" initiative or part of their standard paramedic role (Table 2, quote 1e). 
Table 2. Participant quotes supporting the themes of (1) role confusion, (2) inadequate knowledge base, (3) inadequate feedback, (4) undefined accountability, and (5) patient advocacy.

(1) Role Confusion

1a "It's part of the doctrine of EMS. Everything is very focused on getting the patient on your bed and off to the hospital; they time you." - Participant 101

1b "If I work with another person and I want to refer to CREMS and I say to my partner, 'Hey can you bring the CREMS kit in,' they go: 'What?' It's a clear plastic envelope usually... but you have to hunt for it, you have to find it. Usually it ends up buried. It's literally and figuratively buried in the truck, and buried in the mindset, because the culture is still very focused on beating a stop watch and get patients to the hospital."

- Participant 101

"Emphasize it more. It's not really emphasized at all. Until this past CME, I haven't heard about it from anyone." - Participant 122

1c "Medics have trouble buying into anything new, so if they aren't getting any feedback, if they are not seeing any difference, they are generally just going to think there is no point to it. They are just going to quit, very quickly. 'Well, screw this, I'm not doing this. 'It's similar to when we do clinical trials with drugs, you always get the well oh I don't want to do this, one more drug to carry, when are we going to get a pay increase for this, are we going to get three more percent in the next contract? It always seems to come back to that for a large portion of the profession. And I think that that same sort of mindset is some sort of barrier to some medics. I don't see the benefit in doing these CREMS referrals, so why would I do it?" - Participant 109

1d Interviewer: "So you wouldn't consider CREMS part of that community paramedicine umbrella?"

Participant 103: "I would say... its more of a social service.... than it is necessary community medicine. I don't feel like it's practicing medicine in any way, I feel like it's more of a I'm distributor of information for your resources...I don't feel like I'm really helping in that way. It's not like I'm coming in there and being like, 'Well you can use a lift, or something, you can use, oh, a bath tub, we can put a handle in over here.' I'm not doing any of that, somebody else is doing it. I can refer you to somebody else that can do it. So I don't feel like we are necessarily part of that." Interviewer: "Is CREMS part of community paramedicine?"

Participant 101: "Maybe. I don't know. It's probably... it's got to be related somehow. It's outside of the traditional box or dogma of showing up, giving some sort of treatment and running someone off to the hospital. So I guess anything that is outside of that box shares a community paramedic element."

1e "Sometimes our calls are checking on people...so I guess in some ways we are doing a lot of social work, those calls where it's a check on this person, we do lots of that. But that's under the whole umbrella that is our job, it's not always flying down the street running to emergency calls. A lot of times it's helping people off the floor in the middle of the night. Perhaps they live alone, or in some situation their spouse is unable to assist them." - Participant 110

"Stop the notion that every patient needs to go to the hospital. We get a lot of feedback on cancelled calls and I think that we need to embrace that skill set to not take everyone to the hospital. We are going to the front line and seeing patients in the state they are living in. We are trained to play that role and I hope we do in the future." - Participant 114

(2) Inadequate Knowledge Base

2a Interviewer: "Are you clear on the process of the CREMS program?"

Participant 101: "No."

Interviewer: "What are you unclear about?"

Participant 101: "What exactly happens once we refer a patient. How long it takes once they are contacted by CCAC and what happens once they are contacted. I assume, and this is an assumption on my part, that somebody will come into the home and do a needs assessment, and work with the client to develop strategies to keep them safe at home. I am not a hundred percent sure, and that is because no body has told us. No idea. I am unclear on how I could best handle situations where patients are receiving CCAC services but they need to be redefined or re-examined. Maybe I am encountering someone in crisis and I think that information needs to be communicated to CCAC. So I am not really sure the appropriate channels to go through for that."

$2 \mathrm{~b}$ "There is absolutely no dialogue happening now and it's not anyone's fault... maybe it's the services' fault because they just put this information out on vehicles without providing a concept of use. There is no training; there is no instruction; no instructional manual on how to use this. It's: here's these forms." - Participant 101

2c Interviewer: "Would you explain the CREMS program in your service?"

Participant 104: "The CREMS in my service is... I guess just if you find people who are at risk, in their homes, and get them in touch with the people that can help them, right, like CCAC, or if they need extra services."

Interviewer: "Who would you identify as at risk?"

"Usually people who are a little bit older, maybe they have some mental health issues that prevent them from taking care of themself on their own. Or the older couples, where there may be two of them, but maybe no family to help them out." - Participant 104

$2 \mathrm{~d}$ "I don't think I have a thorough understanding of how exactly they [CCAC] can help when I make the referral. I kind of make it up when I'm talking to somebody." - Participant 107

2e Interviewer: "Why do you feel you are unsure about those things [which patients to refer to CREMSI?"

Participant 101: "Lack of training, for sure. Training would be very helpful. One, I would know what services are offered, two, I would know what services are available for people, third, I would understand the referral process a little bit more, and four, I would have a mechanism to do some follow up in a crisis situation." 


\section{Table 2. (Continued)}

Interviewer: "Does that affect who you refer?"

Participant 101: "If anything I think I refer more people than I should."

Interviewer: "Because you don't understand the program?"

Participant 101: "Yeah...because maybe I am over-referring people. Maybe I am referring candidates that are not appropriate candidates for the service."

$2 f$ "It is my obligation as a paramedic. I feel that there are people who are falling through the cracks, who aren't getting the help they need. I guess, like I said prior to, if I think that this person, yeah, could benefit from the referral program, and they aren't necessarily a drain on the system, I feel like they should be enrolled, because something good could come out of it. I feel like there are a lot of regulars [referring to frequent users of EMSI that wouldn't benefit from it and end up costing us more money, and just looking at the cost-benefits of it, its not necessarily worthwhile for me to enroll someone into the program or suggest to enroll them into the program because it's just not going to help that person. Again, I realize that it's not for me to make that decision, but sometimes that is how I choose and not choose to enroll people." - Participant 106

$2 \mathrm{~g}$ "So I would say experience really. And you see the same thing over and over again, and they bring in the CREMS program to help, and it's sort of an extra thing that can sort of help. It helps you recognize who needs help. Right, so I think it's with experience that when you see these calls over and over again and you learn to look at those surroundings, right. And when you are new you are still focused on those patient care things because you haven't had the experience." - Participant 119

\section{(3) Inadequate Feedback}

3a "Medics, I think, will do things really well and will work really hard, provided they get the feedback. Right, if they are just doing things and the actions are just going off into the abyss, into the void, and they don't get any feedback of what they did was right, or wrong, or effective or not effective, eventually they stop. So I think feedback would be a welcome initiative, at least for me." - Participant 101

3b Interviewer: "What are the outcomes of CREMS?"

Participant 101: "Hopefully to recognize the needs of elderly patients that are residing at home, any gaps in their care, and learning the CCACS and how CREMS addresses these issues to get what they need."

Interviewer: "Do you think you are accomplishing those objectives?"

Participant 101: "Again I don't know. Because I don't have any reason to know if these referrals are doing anything. I don't have any feedback or information to reflect either way."

3c "I don't know if they work well period. That's the trick. I don't know if this CREMS thing actually works. I know it works from my end, that I establish rapport with my patients, and I fill out this referral, and I put it in the envelope and it gets sent off and I get an email that says thank you we got your form. But after that, I have no idea if it is effective, not a clue." - Participant 101

"We don't really get a whole lot of feedback as to what happens afterwards, so I am assuming that it is working. That's why we keep referring people." - Participant 102

"That's what I mean about not being able to get anything back. I mean you get that email saying, 'Thank you for your CREMS referral, ' but I don't know what they got, so I don't know if anything is there. I certainly have put in patients with a CREMS referral before and have gone back to that same person and nothing has happened. So based on that, I just feel like nothing has happened." - Participant 106

\section{(4) Undefined Accountability}

4a "I wouldn't say I chose not to out of maliciousness, I would say this person is a CREMS referral and I would certainly do it, but then I get a call, and then I get another call and you don't get that opportunity to sit down there and write it up and do that phone call. And eventually it just falls right out of your head, and I might think about it at the end of the shift, but I'm too tired and I'll try to do it the next day, but eventually it just falls right out of my head and forget about it until I find that piece of paper in my pocket when I'm doing my laundry and I feel like it's too late at that point." - Participant 106

$4 \mathrm{~b}$ "With the referrals, you never know the outcome. You never know if anyone ever falls out. EMS is a large service, especially the service I work for... because things get lost in the shuffle so much and I'm so used to seeing that, I feel like the CREMS referrals never got to where it's supposed to go and if it did the person that is responsible for it is either perhaps a light duties person or someone who isn't invested in the program. They don't really care if it's going to go forward or not. And that's a little frustrating that you never know where it goes, so it's questionable if it goes anywhere at all." - Participant 106

\section{(5) Patient Advocacy}

$5 a$ "You know, you hate going in to someone's house that you feel so bad for because they live by themselves and they have nobody to help care for them, and they are older. You just want to get them the help that they need." - Participant 102

5b "My primary focus is what is better for the patient, both in the short, medium, and long term." - Participant 101

$5 \mathrm{c}$ "Especially if I know that patient is not really going to receive proper definitive care in emergency [department], and [the ED] doesn't necessarily see what we had in the home that led me to think that this person needs more help, they will treat the chief complaint and maybe send them home and not be addressing the underlying cause if the patient does not bring it up. So having seen it first person in the patient's home environment, it's a better way for me to care for them" - Participant 109 
Referral programs are at the intersection of two competing ideologies, creating challenges and a potential conflict for paramedics, given their dominant and well-established "emergency" role. In our interaction with frontline paramedics and through identification of additional themes discussed next, we recognized how the theme of conflicting roles was likely more robust than we first appreciated. Elements of training, communication, accountability, and patient advocacy all intersect and highlight the importance of role changes in EMS as the trend continues to include preventative and community care.

\section{Inadequate knowledge base}

Participants expressed a limited understanding of referral programs, factors supporting referral programs, and skewed perceptions of patient eligibility despite most participants having received some form of education. Participants shared indicators, and at times expressed directly, that the education they received may have been inadequate to prepare them for the task of referring patients (Table 2, quote 2a). When asked specifically about the education they received, it was clear that significant variation existed, with some receiving training by continuing medical education, email communication, or none at all (Table 2, quote $2 b)$. Some expressed uncertainty that they were adequately referring all patients (Table 2 , quote $2 \mathrm{e}$ ). As a result, frontline paramedics had highly subjective and disparate views regarding appropriate patients for a referral, the types of services that might be available to patients, and in a few cases, the organization to which they would refer patients. When participants discussed the types of patients they referred into the program, responses were typically vague and included, at times, a simple emphasis on older adult patients (Table 2, quote 2c). When discussing the referral program, "I think" and "I assume" were common in the dialogue. Some "made it up" while talking to patients about the program (Table 2, quote 2d). For some, decisions regarding whom to refer were based on their personally held beliefs regarding who was eligible for, or required, care (Table 2, quote 2e). Furthermore, participants did not attribute the skills used to identify patients eligible for referral to education, but to experience with certain patient groups. Participants with more years of experience commented on the benefits of experience when considering patients for referrals (Table 2, quote $2 \mathrm{f}$ ).

\section{Inadequate feedback}

Participants reported receiving feedback from their respective services following a referral; however, the lack of timeliness and the limited details often rendered the feedback unreliable and at times meaningless (e.g., made it difficult to improve performance and accuracy, and to reinforce the program's success) (Table 2, quotes 3a, 3b). At times, feedback did not occur by design, but instead occurred as a result of seeing the same patient on multiple occasions. Participants who reported that they had observed patients who had received services following a referral expressed positivity towards the referral program and its contribution to patients' wellbeing. In contrast, participants who reported returning to patients' homes and perceived little or no change in service provision, or who observed patient deterioration, expressed negative views of the referral program (Table 2, quote $3 \mathrm{c}$ ). It is worth noting that although participants stated that they rarely, if ever, received feedback from patient interactions they experienced on a daily basis, receiving feedback from a referral remained an important element of the program.

\section{Undefined accountability}

The concept of accountability emerged in a number of ways. Participants felt that they themselves were not held accountable by the program, and that perhaps no one was. At the time of this study, it was not mandatory for paramedics to refer patients and therefore completing referrals was also not recognized as a core responsibility (Table 2, quote $4 \mathrm{a}$ ). The perceived lack of accountability by the service was reinforced when participants expressed uncertainty that their referral was ever acted upon by their EMS service or CCAC. Participants often described their referral moving "off into a black hole," with the "hope" that someone would provide care to the patient (Table 2, quote $4 \mathrm{~b}$ ). Combined with a lack of knowledge of the program, and the absence of meaningful feedback, participants often expressed that no one, including the employer, and in some instances CCAC, were accountable to the program. For some participants this resulted in a decrease in their use of the program.

\section{Patient advocacy}

Many participants expressed that referral programs were a mechanism that allowed them to enhance their 
role as patient advocates, often serving as a motivator for participating in the program. Participants acknowledged the importance of their role in providing long-term additional assistance for patients, and that it was their obligation to help (Table 2, quote 5 a). Some participants expressed uncertainty about whether informal reports to hospital staff upon transfer of care were having the intended effect; thus, having a formal referral method filled a much-needed void (Table 2, quotes 5b, 5c). Participants' sense of their role as patient advocates was robust. Participants expressed "hope" in the health care system and that the patients' needs would be appropriately addressed. This was a common response among participants, suggesting that patient advocacy often replaced or at least mitigated many of the challenges discussed prior.

\section{DISCUSSION}

This study explored the experiences of frontline paramedics with referral programs and identified: (1) an incomplete integration of referral programs into what paramedics identify as their role, (2) inadequate education to support the programs, (3) an incomplete infrastructure to support paramedic development in this area, and (4) a perceived absence of accountability with referral programs. However, the study also identified a strong and seemingly ingrained (5) commitment to patient advocacy that may provide a meaningful platform by which to support referral programs.

The introduction of referral programs appears to represent a departure from how many frontline paramedics define their role in the health care system. Paramedic identity appears to be influenced by frequent implicit and explicit messages that paramedics receive. Evidence of this limited or incomplete integration of the CREMS program into paramedic practice may contribute to implementation challenges of community-based care experienced by the profession. However, a reframing of paramedic practice is under way worldwide ${ }^{10}$ and likely to become increasingly present. Still, this study suggests that incongruent messages impacted how paramedics integrated this relatively new role into their professional identities, and ultimately their patient care.

Further, optimizing referral programs may remain challenging without improving educational strategies to support them. We identified numerous direct and indirect references to limited knowledge and understanding regarding a number of elements believed to be vital for referral programs, and participants linked these deficiencies to inadequate education. Indeed, others have identified limited education in this area at the entry to practice and ongoing professional development levels, ${ }^{11}$ and the need for further or better training to improve accuracy or appropriateness in referral programs $^{3}$ and screening. ${ }^{5}$

Another salient theme emerging from the data was the strong desire by paramedics to receive meaningful feedback and improve communication regarding referrals made. In cases where feedback lacked detail or was delayed it did little to improve paramedics' use or understanding of the CREMS program. Despite many challenges that likely exist, (e.g., privacy legislation, logistical challenges), the paramedic and health care community will need to find innovative strategies to optimize feedback, or place greater emphasis on continuing education and make clear what paramedics can expect and why.

In addition, there appeared to be a perception by frontline paramedics that there was a lack of accountability for referral programs at any level. On an individual level, a missed opportunity to report a referral was seldom identified. Likewise, when a referral was made, it was unclear who was responsible for the referral and what quality indicators were in place to ensure appropriate and timely follow-through. When referrals were missed without consequence, or when referrals seemed to disappear once made, this may have further reinforced the perceived lack of accountability to the program and resulted in questions regarding its relevance.

Despite these challenges, the seemingly engrained concept of patient advocacy may serve as an opportunity to support referral programs going forward. Referral programs appear to fill a void for both paramedics and patients, and therefore represent a clear opportunity. However, many of the challenges discussed above compete with or are in conflict with this desire to serve patients. Still, paramedics recognize patient advocacy as a fundamental role in their daily practice.

\section{Implications}

By examining the incorporation of community health initiatives through the perspective of paramedics, this study illuminates the complexity and robustness of 
paramedic culture and operations and its potential impact on community-based programs. This exploratory study highlights the need for intervention and future research on how paramedics' role and identity are changing as EMS continues to include or expand community paramedicine initiatives. This study identified educational difficulties as part of this change, suggesting that greater emphasis on initial and ongoing educational strategies should be explored. Future research should also address whether the current lack of education about the program has resulted in hesitation to participate in the program, missed opportunities, or inappropriate referrals, leading to an ineffective program. Furthermore, we recommend that EMS implementing a referral program, or those with current programs, take a paramedic-centered approach, and consider factors such as accountability to the program (such as implementing mandatory referrals and a clear explanation regarding who is responsible for the program) and a method to address feedback (or explain why it is not possible).

\section{LIMITATIONS}

It is important to note the limitations to this study. First, only one participant reported making zero referrals. One possible explanation for this is that paramedics who are interested in CREMS would be more likely to participate in the study, compared to those who are not. Thus, identifying specific barriers to engaging in CREMS programs will require additional research. Furthermore, due to limitations in our initial enrollment strategy, we adopted a snowball sampling strategy. Snowball sampling is a common strategy in qualitative research, but can be associated with an increased risk of bias. However, only seven of the 23 participants were contacted using this method. Furthermore, this group ranged in their perceptions of the program, years of experience, and reported referrals. Lastly, this exploratory qualitative study did not attempt to make generalizations about population, but rather exposed key concepts worth exploring in more detail.

\section{CONCLUSION}

In summary, the implementation of referral programs, while grounded in a need and opportunity to serve patients in a constrained health care system, presents challenges and opportunities for the paramedic profession. While paramedics view referral programs as a welcome opportunity to address patients' needs in their everyday practice, this new role has not yet been fully incorporated into the profession. The success of referral programs may be limited if role confusion, inadequate knowledge and understanding, opportunities for continued development, and accountability indicators are not adequately addressed.

Competing Interests: None declared.

\section{REFERENCES}

1. Sinha S. Living Longer, Living Well: highlighting and key recommendations. From the Report Submitted to the Ministry of Health, and the Minister Responsible for Seniors on recommendations to inform a Seniors Strategy for Ontario. Available at: http://www.health.gov.on.ca/en/ common/ministry/publications/reports/seniors_strategy/docs/ seniors_strategy.pdf (accessed January 2013).

2. Denton M, Ploeg J, Tindale J, et al. Would older adults turn to community support services for help to maintain their independence? I Appl Gerontol 2009;29(5): 554-78.

3. Kue R, Ramstrom E, Weisberg S, et al. Evaluation of an emergency medical-services based social services referral program for elderly patients. Prehosp Emerg Care 2009;13(3): 273-9.

4. Mann N, Hedges JR. The role of prehospital care providers in the advancement of public health. Prehosp Emerg Care 2002; 6(2):S63-7.

5. Shah MN, Caprio TV, Swanson P, et al. A novel emergency medical services-based program to identify and assist older adults in a rural community. 7 Am Geriatr Soc 2010;58(11): 2205-11.

6. Creswell JW. Research Design: Qualitative, Quantitative and Mixed Methods Approaches. Thousand Oaks, CA: Sage Publications; 2009.

7. Charmaz K. Constructing Grounded Theory: A Practical Guide Through Qualitative Analysis. Thousand Oaks, CA: Sage Publications Ltd; 2006.

8. Morse JM, Barrett M, Mayan M, et al. Verification strategies for establishing reliability and validity in qualitative research. Int 7 Qual Methods 2002;1(2):13-22.

9. Bryman A, Teevan JJ, Bell E. Social Research Methods, 2nd ed. Toronto, ON: Oxford University Press; 2009.

10. Evans R, McGovern R, Birch J, et al. Which extended paramedic skills are making an impact in emergency care and can be related to the UK paramedic system? A systematic review of the literature. Emerg Med 7 2014;31(7):594-603.

11. O'Meara P, Ruest M, Stirling C. Community paramedicine: higher education as an enabling factor. Aust 7 Paramed 2014;11(2):5. 